\title{
La interacción de identidades en América Latina y su influencia en la política exterior sobre las drogas*
}

\author{
Luz Aurora López Flórez** \\ (luz.lopez0789@gmail.com)
}

Artículo de revisión recibido el 27/11/2013 y aprobado el 27/02/2014.

\begin{abstract}
Cómo citar este artículo:
LÓPEZ FLÓREZ, Luz Aurora (2014). "La interacción de identidades en América Latina y su influencia en la política exterior sobre las drogas”. En: Trans-pasando Fronteras, Núm. 5, pp. 93-107. Cali, Colombia: Centro de Estudios Interdisciplinarios, Jurídicos, Sociales y Humanistas (CIES), Facultad de Derecho y Ciencias Sociales, Universidad Icesi.
\end{abstract}

\begin{abstract}
Resumen
Hechos recientes han demostrado que las políticas represivas para contrarrestar el problema de las drogas están siendo cuestionadas en América Latina. Es por ello que este artículo pretende, desde una perspectiva constructivista, responder a la pregunta ¿en qué medida la existencia de un cambio en la percepción del fenómeno de las drogas, ha generado un giro en las políticas destinadas al control de las mismas en América Latina?
\end{abstract}

Palabras clave:

Identidad, América Latina, política exterior, constructivismo.

* La identidad se entiende como "es el proceso por el cual los actores sociales construyen el sentido de su acción atendiendo a un atributo cultural [social, económico o político] (o conjunto articulado de atributos) al que se da prioridad sobre otras fuentes posibles de sentido de la acción" (Castells, 1999).

** Estudiante de Ciencia política en la Universidad Icesi. 
La economía desarrollada alrededor de los estupefacientes, desde hace varias décadas, hace parte desde hace varias décadas, de la agenda de los gobiernos a nivel mundial, no solo a nivel nacional sino a nivel internacional. Esto se debe a que, en tanto que economicamenteeconómicamenteía, las drogas implican una serie de procesos productivos, de comercialización y consumo que involucran a una gran cantidad de agentes alrededor del mundo. Debido a lo lucrativo de su negocio y su desarrollo en un marco de ilegalidad, el tema de los estupefacientes ha sido desde hace medio siglo un eje de cooperación a nivel internacional. América Latina, como principal proveedor de cocaína, ha tenido un gran interés ser parte de este ámbito de cooperación en la adopción de políticas que permitan disminuir los efectos negativos ocasionados por la economía de narcóticos.

La globalidad de esta economía, unida a al "la creciente "densidad dinámica" (Wendt, 2005:20) [2] que enfrentan los países en todo el mundo, justifica los incentivos generados por la cooperación en la llamada lucha contra el narcotráfico. Bajo la perspectiva de una búsqueda de ganancias colectivas, se han creado numerosas organizaciones, nacionales e internacionales, que trabajan para reducir los efectos negativos generados por el negocio de estupefacientes, en todos los niveles: producción, consumo y comercialización. Igualmente se han firmado tratados y se han realizado cumbres para discutir todo tipo de temáticas entorno al problema de las drogas. En la VI Cumbre de las Américas, realizada en Cartagena en abril del 2012, el foco central era "el rol de la integración fisica y la cooperación regional, como medio para alcanzar mayores niveles de desarrollo y superar los desafios del hemisferio en varias áreas claves incluyendo la pobreza y desigualdades, seguridad ciudadana, desastres y acceso y uso de tecnologías" (Secretaría OEA). En este marco, uno de los pronunciamientos realizados por los jefes de Estado participantes, fue el apoyo a la realización de Conferencia Internacional de Ministros de Relaciones Exteriores y de Jefes de Organismos Nacional Especializados contra el Problema Mundial de las Drogas en junio de 2012 en Lima, Perú, y la participación activa en dicha conferencia (OEA, 2012).

El discurso que se expone en este comunicado y que justifica la realización de la conferencia mencionada, es evidencia de los valores que rigen a la comunidad de Estados americanos. Además, muestra la preocupación de los diferentes gobiernos por un refuerzo de las medidas aplicadas a la solución del "problema mundial de la droga", refuerzo que como se observó en varios de los discursos de los presidentes, está encaminado a una revisión de 
estas medidas que mejoren su eficacia. En este sentido, este comunicado es igualmente un ejemplo del efecto de las identidades sobre el proceso de toma de decisiones en la política exterior de los Estados. Además, hechos recientes muestran que las políticas represivas para contrarrestar el problema de las drogas están siendo cuestionadas en América Latina. Es por ello, que desde una perspectiva constructivista, es válido preguntarse ¿en qué medida la existencia de un cambio en la percepción del fenómeno de las drogas, ha generado un giro en las políticas destinadas al control de las mismas en América Latina?

\section{El régimen de la lucha contra las drogas}

La construcción de significados colectivos es uno de los elementos esenciales en la construcción de identidades, pues "los actores adquieren identidad - expectativas e interpretaciones del yo relativamente estables y acordes con su papel- mediante su participación en estos significados colectivos" (Wendt, 2005:6). Estos significados son construidos tanto en el ámbito nacional como internacional. A nivel internacional, parte de la construcción de estos significados colectivos reposa sobre los diferentes documentos que reglamentan el control de los estupefacientes. Estos documentos han sido diseñados por las Naciones Unidas, que es la organización formal de Estados más importante a nivel mundial, pues es quien define gran parte de los parámetros que los Estados en el mundo deben seguir. El régimen internacional que define la reglamentación frente a las drogas tiene sus inicios en la Convención única de 1961 sobre los estupefacientes, posteriormente esta Convención es enmendada por el Protocolo de 1972. A esta le suceden el Convenio sobre sustancias sicotrópicas de 1972 y la Convención de las Naciones Unidas contra el tráfico ilícito de 1988 (Hopenhayn y Arriagada, Octubre 2000).

La Convención única de 1961 sobre estupefacientes (Naciones Unidas, 1961), define la manera como se debe realizar el control de los estupefacientes relacionados al opio, coca y cannabis de acuerdo a la lista que le ha sido asignada según sus características, respecto a su uso científico, médico o ilegal. Igualmente designa a la Comisión de Estupefacientes del Consejo Económico y Social y a la Junta internacional de Fiscalización de Estupefacientes como los órganos internacionales de fiscalización, y define sus funciones y funcionamiento. El Convenio sobre sustancias sicotrópicas de 1972 (Naciones Unidas, 1972) regula el control de sustancias como las anfetami- 
nas, barbitúricos, benzodiacepinas y las substancias psicodélicas, las cuales habían sido descubiertas recientemente. Finalmente, la Convención de las Naciones Unidas contra el tráfico ilícito de 1988 (Naciones Unidas, 1988) busca promover la "cooperación entre las Partes a fin de que puedan hacer frente con mayor eficacia a los diversos aspectos del tráfico ilícito de estupefacientes y sustancias sicotrópicas que tengan una dimensión internacional" (Naciones Unidas, 1988), cumpliendo con las obligaciones definidas en la Convención, de acuerdo con los ordenamientos jurídicos internos. En esta medida, regula las acciones que se deben tomar en torno al tráfico ilícito por diferentes medios e implementa regulaciones sobre las materias primas que permiten la producción de sustancias ilícitas.

El Latinoamérica, a presente, todos los Estados son parte de la Convención de 1988 (Junta Internacional de Fiscalización de Estupefacientes, 2013). Honduras y Santa Lucía aún no son parte de la Convención de 1972, Haití aún no se ha unido a la Convención de 1971, Guyana no hace parte de la Convención de 1961 y Nicaragua no pertenece a la forma enmendada de la Convención de 1961. Por otra parte, Belice, San Vicente y Granadinas no hacen parte ni de la Convención de 1961, ni la de 1972. Estos datos muestran una adherencia general de los países latinoamericanos al sistema de normas y valores implementado por las Naciones Unidas respecto al control de estupefacientes. De los tres documentos nombrados, podemos deducir que el sistema de normas y valores ${ }^{2}$ propuesto es el siguiente:

- Normas: control de las sustancias mencionadas en las diferentes convenciones (opio, cannabis, coca, anfetaminas, barbitúricos, benzodiacepinas y las substancias psicodélicas); suministro de la información concerniente a la producción, fabricación, uso, consumo, importación, exportación, decomiso, existencias, superficies de cultivo de los diferentes estupefacientes; rendición de cuentas; alineamiento de políticas internas y lineamientos de las convenciones; cooperación entre las partes; ejercicios de acciones preventivas y represivas (castigos, privación de la libertad, penas de prisión) contra quienes cometan actos delictivos concernientes a los estupefacientes y sustancias psicotrópicas, puesta en marcha de tratamiento y reinserción social para consumidores (sugerido). 
- Valores: la cooperación entre los Estados, el respeto por los derechos humanos y la dignidad humana, respeto de la igualdad soberana, de la integridad territorial de los Estados, la no intervención en los asuntos internos de otros Estados, la conservación de la civilización.

La adherencia a las convenciones mencionadas, es para los Estados una muestra de aceptación de las reglas de la comunidad internacional y por tanto, la adhesión a las mismas prueba su pertenencia a esa comunidad. Y a partir de estos documentos, podemos identificar el significado que tiene para la comunidad internacional el mercado de las drogas. El preámbulo de cada uno de los convenios, parte de la expresión de la inquietud de las Partes (países adherentes) sobre la "salud fisica y moral de la humanidad" (Naciones Unidas, 1961) y "la salud y bienestar de los seres humanos" (Naciones Unidas 1972 y 1988); a lo que siguen afirmaciones que indican que el indebido manejo de los estupefacientes atenta contra esa salud y bienestar de la humanidad. Estas afirmaciones son evidencia de la concepción negativa que se tiene de todas aquellas sustancias que tienen actúan sobre el sistema nervioso central, y que generan cambios temporales en el estado de conciencia de las personas, sus estados de ánimo, su comportamiento y/o la percepción de su entorno.

De hecho, esta percepción negativa tiene origen en las implicaciones a nivel social que tiene la afectación de la psiquis de las personas. Estas implicaciones son consideradas como un elemento que socavaba los cimientos de la sociedad (Naciones Unidas, 1988:1), lo cual ha puesto a estas sustancias en el lugar de un enemigo para la sociedad al que ha sido necesario declararle la guerra. "La guerra contra las drogas ”, es una expresión que se popularizó después del discurso pronunciado el 17 de junio de 1971 en Washington por el expresidente de los Estados Unidos Richard Nixon y sigue vigente hasta el día de hoy. De hecho, en este discurso (Nixon, 1971), Nixon caracteriza a las drogas como un destructor de vidas, familias y comunidades, ya que están relacionadas con un alto índice de mortalidad, violencia y crimen. Es por ello que en este discurso Nixon muestra su compromiso con la prohibición de las drogas psicoactivas y anuncia una serie de proyectos para atacar el problema a nivel interno y externo, que incluye programas de rehabilitación para los consumidores estadouni- 
denses y aumento del pie de fuerza para reprimir a los vendedores de drogas, al igual que paquetes de ayuda militar para los países donde se realizaba de la producción y exportación de este tipo de sustancias.

Esta aproximación preventiva y represiva, es recurrente en los Convenios mencionados. Su firma, compromete a las Partes a inscribirse en esa lucha contra las drogas y por tanto implica la realización de acciones que estén encaminadas a participar en esta lucha. De hecho, en el artículo 20 de la Convención de 1988, compromete a las Partes a proporcionar "el texto de las leyes y reglamentos que promulguen para dar efecto a la Convención” (Naciones Unidas, 1988), lo cual garantizaría el alineamiento de las leyes nacionales con dicha convención. Además, afilia a estos países a percibir el fenómeno de las drogas, económicamente hablando, desde la Ley de Say que expresa que la oferta crea su propia demanda. Las implicaciones que esto tiene para la identidad de los países en Latinoamérica son considerables.

A partir de esta perspectiva, se define a los productores y exportadores de drogas ilícitas como los responsables de los problemas sociales, políticos y económicos generados por el consumo de drogas. Esta caracterización, aplica a algunos países latinoamericanos en la medida en que se establecen como los principales productores de coca a nivel mundial, e incluye a otros países del continente al introducirlos el sistema de fabricación, comercialización y consumo de estupefacientes, al igual que en el proceso de lavado del dinero obtenido de las ganancias del negocio de narcóticos. El rol de productores, genera un reconocimiento por parte de la comunidad internacional de la alta responsabilidad que tienen los países latinoamericanos en la disminución del problema enfrentado a nivel mundial por las drogas (Kaplan, 1993). Esto se refleja en las ayudas recibidas por los países latinoamericanos en su lucha contra las drogas por parte de la comunidad internacional y especialmente por parte de Estados Unidos.

A pesar de los esfuerzos militares realizados para enfrentar el tráfico de drogas, el enfoque utilizado, lejos de aportar soluciones al problema ha generado diversos conflictos. Ante la búsqueda de una disminución del tráfico, en los informes presentados ante las Naciones Unidas se observa que entre 1995 y el 2005 tanto las incautaciones de cocaína como de hierba de cannabis aumentaron considerable. En un plazo de diez años, las incautaciones de cocaína se duplicaron y las de hierba de cannabis aumentaron en un $50 \%$. 


\section{Gráfico 1}

Incautación mundial de cocaína, 1995-2005

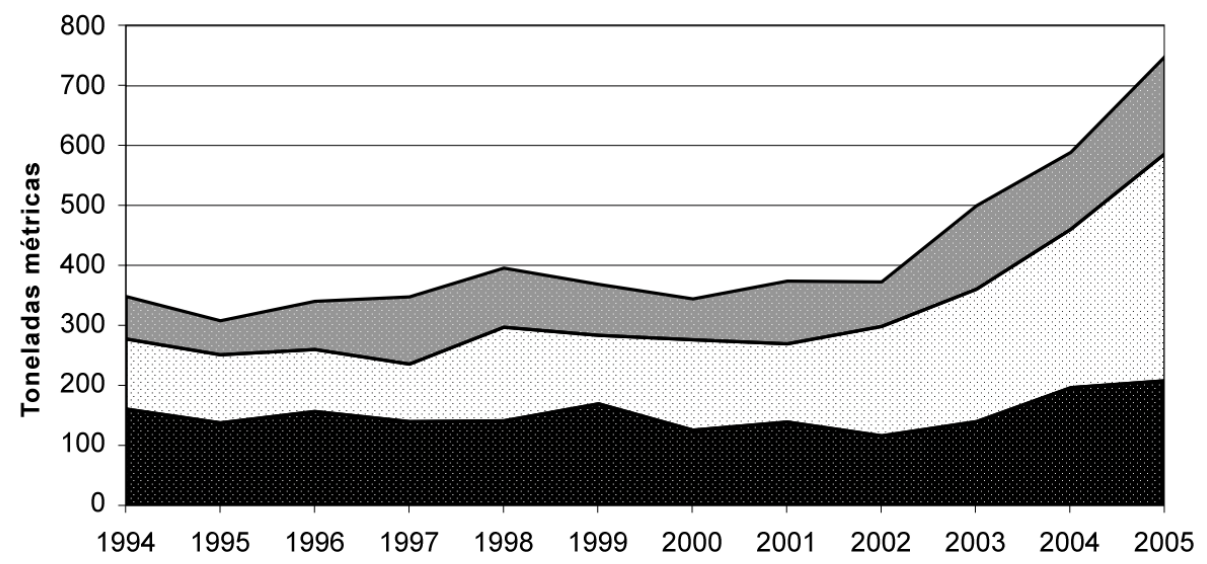

América del Norte $\square$ América del Sur $\square$ Otros

\section{Gráfico 2}

Incautación de hierba de cannabis en América y a nivel mundial, 1995-2005

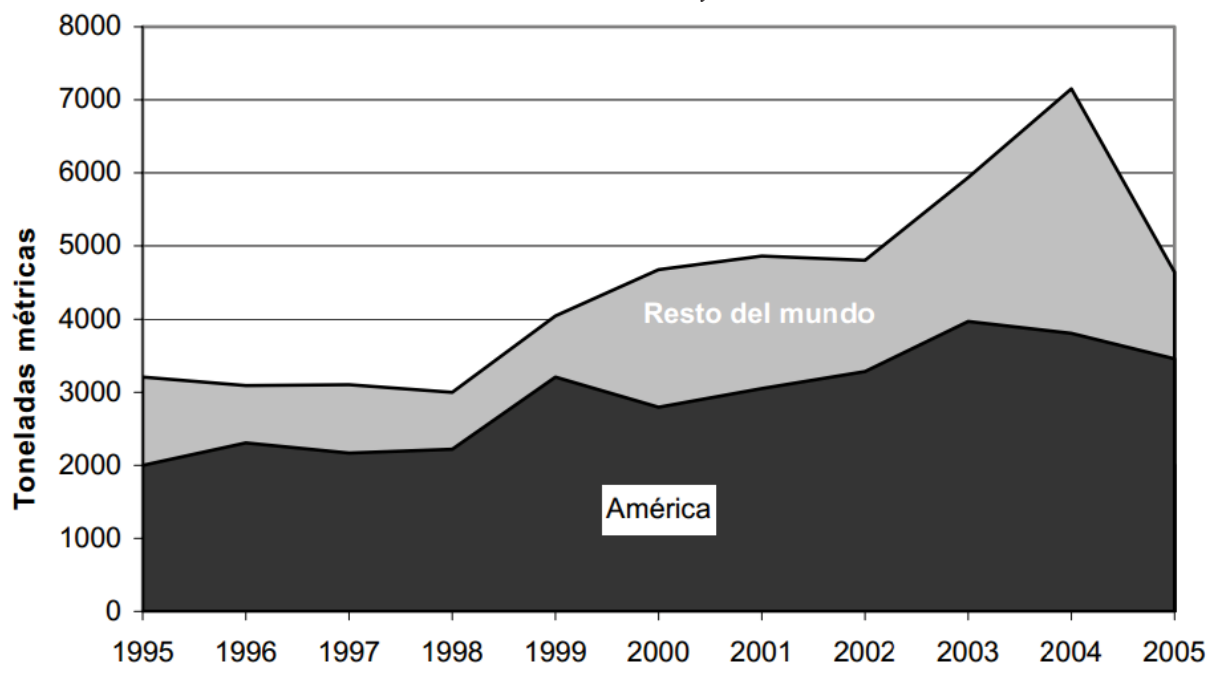

Fuente: Principales tendencias regionales del tráfico de drogas y medidas para combatirlo. 12 de agosto de 2007. 17 Reunión de Jefes de los Organismos Nacionales Encargados de Combatir el Tráfico Ilícito de Drogas, América Latina y el Caribe Quito (Ecuador), 15 a 19 de octubre de 2007 Tema 3 del programa provisional. Naciones Unidas Contra la Droga y el Delito. 
Por otra parte, durante los cincuenta años que se han aplicado las leyes de restricción y cero tolerancia, se ha constatado un recrudecimiento de la problemática socio-económica generada en torno al problema de las drogas, respecto a su rol como fuente de ingresos y a los problemas de salubridad respecto a su consumo. Estos son los argumentos señalados por diversos países que proponen la búsqueda de nuevas alternativas en lo que se refiere a las políticas sobre las drogas (Hopenhayn y[6] Arriagada, Octubre 2000).

\section{Transformaciones del sistema de normas y valores frente a la lucha contra las drogas}

Progresivamente, la lucha contra las drogas ha dejado de tener como base la perspectiva de una seguridad entendida como las amenazas militares para la sobrevivencia humana y bienestar, sino que a esto se agregan las amenazas a la salud, al medio ambiente, a la economía y a todas las esferas que involucran a los seres humanos (Kegley y Wittkopf, 2006, pág. 493). Los Estados definen la seguridad "en términos de 'interés propio"” (Wendt, 2005, pág. 1), ese interés propio está dado por la valoración que estos hacen de cada una de las esferas mencionadas. En este sentido, se observa un cambio en las pautas que definen los elementos que componen el interés propio de los Estados en Latinoamérica.

Como se esbozó con anterioridad, hay un contexto socioeconómico que marca la manera como hoy se percibe el fenómeno de las drogas (Hopenhayn y Arriagada, Octubre 2000). En primer lugar, es de gran importancia resaltar que el tráfico de drogas ha generado una serie de externalidades, que hacen que los problemas de violencia y crimen ya no solo estén definidos en términos de oferta y demanda. En la actualidad, las drogas son ampliamente percibidas como un mercado que incluye no solo la producción y el consumo, sino además una red de comercio o tráfico, y de lavado de dinero. De esta manera, países como México, Argentina, Venezuela, Chile, Costa Rica y las islas del Caribe, han tomado un mayor protagonismo en el control del tráfico. Por otra parte, a nivel socio-económico, se comienzan a entender las dinámicas que constituyen a la economía de las drogas como una fuente de ingresos y empleo. A nivel macro, el lavado de dinero es considerado como una de las principales fuentes de ingresos de los países llamados paraísos fiscales, como es el caso de Panamá, Dominica, Bahamas, San Cristóbal y Nevis, San Vicente y Granadinas. A nivel micro, para muchas personas de bajos recursos y alta vulnerabilidad, las drogas constituyen una alternativa de empleo y mejoramiento de sus ingresos. 
Otro de los elementos asociados a esta economía, es la cultura de ilegalidad (Hopenhayn y Arriagada, Octubre 2000) que contribuye a acrecentar la violencia en algunos de los países involucrados en ella y que ha generado problemas de corrupción a nivel de los gobiernos. En una declaración hecha por el expresidente mexicano Vicente Fox, (Lozano, 2012) se muestra la preocupación por la recrudecimiento de la violencia en México. En sus palabras, esto ha deteriorado la imagen del país ante la comunidad internacional y ha cobrado la vida de 60.000 jóvenes en cinco años. Además, recientemente se han aumentado las alarmas sobre la expansión del dominio de los careles mexicanos en los estados del Este de Estados Unidos, quienes han encontrado en la heroína un negocio más lucrativo que el de la cocaína (Saiz, 2013). Por otro lado, nos encontramos hoy ante un momento en el que los Estados y la sociedad civil han hecho esfuerzos para reconocer las dinámicas de corrupción en los gobiernos de países que se han incorporado en la dinámica del narcotráfico. Un ejemplo de ello fueron los esfuerzos realizados por la Iglesia argentina para poner en la agenda de la opinión pública, el tema de la amenaza del narcotráfico, hecho que implicó un reclamo por aumento de las medidas de seguridad en la frontera con Bolivia por parte de la Corte Suprema de Justicia (Rey, 2013).

Asimismo, el consumo sigue siendo una problemática de gran importancia, pues se puede constatar que el consumo de estupefacientes es una práctica que empieza a generalizarse en América Latina (Hopenhayn y Arriagada, Octubre 2000). Bien que no se detecta una especificidad de pertenencia socioeconómica de los consumidores, es evidente la vulnerabilidad a la que se enfrentan los consumidores de menores recursos respecto a los daños ocasionados por ese consumo. Esto se debe a que su condición socioeconómica ligada a la criminalización del consumo, hacen que la posibilidad de tratamiento disminuya. Hecho al que se suma la vulnerabilidad psicológica que muchas veces enfrentan debido a las condiciones de violencia y pobreza de su entorno, y constituyen las drogas como una salida de escape. De esta manera, es importante agregar que las dinámicas sociales y las políticas represivas, generan procesos de exclusión y marginalización que empeoran la condición de estas personas.

Ante este panorama, los países latinoamericanos han expresado su preocupación por la búsqueda de nuevas alternativas para redefinir las políticas de las drogas. Un hecho importante que permite observar ese cambio de perspectiva es la, ya mencionada, VI Cumbre 
de las Américas realizada en abril del 2012. Uno de los temas tratados en esta Cumbre fue la posibilidad de una reevaluación de las políticas de la lucha contra las drogas, tema que posicionó dos bandos: aquellos que consideraron ver en la legalización una posible salida al conflicto generado por las drogas, y aquellos que se opusieron a ver en esta una solución viable. En el primer bando encontramos a países como Colombia, Uruguay, México, Brasil, Guatemala y Chile. En el segundo bando, se posicionaron EEUU, Venezuela, Perú y Paraguay. En palabras del presidente colombiano, Juan Manuel Santos, la discusión sobre una posible revisión de la política antidrogas fue un gran paso para la región, en la búsqueda de soluciones más efectivas y menos costosas. En este orden de ideas, declaró que la legalización como una estrategia conjunta podría ser una opción viable (Infobae, 2012). Por otra parte, el presidente de los Estados Unidos, Barack Obama aunque no se negó a participar en el debate, dejó claro que en pro de la salud y la seguridad de sus habitantes la legalización no es una opción para su país (Infobae, 2012).

Recientemente, estos pronunciamientos se vieron enfrentados a un hecho tangible de este debate. El 31 de julio del 2013, la Cámara de representantes de Uruguay aprobó el proyecto de ley que pretende legalizar la venta y cultivo de marihuana. Diversos actores se pronunciaron al respecto. En Uruguay la opinión pública se encuentra dividida. De hecho, el 63\% de los Uruguayos están en contra del proyecto de ley, pues lo consideran una amenaza ante la posibilidad de la legalización del cannabis induzca al consumo de drogas más duras (Oppenheimer, 2013). Por otra parte, en el momento en que se presentó la propuesta, la Casa Blanca expresó sus críticas y la ONU pronunció una advertencia ante la posibilidad de violación de la Convención de 1988. Venezuela expresó su crítica al definir este hecho como una trampa para la seguridad, Argentina y Brasil expresaron su preocupación respecto a los efectos de la medida sobre los países vecinos y Santos criticó la unilateralidad de la medida (El Tiempo, 2012).

Este panorama, refleja claramente un cambio en la manera como los países latinoamericanos se están relacionando con el tema de las drogas. Se comienza a dejar de lado la criminalización del consumo y la perspectiva del consumidor como un ser enfermo que necesita atención por parte del paternalismo estatal (Kaplan, 1993). También, observamos un distanciamiento de los ejes establecidos por la hegemonía estadounidense, al construir bloques de oposición a la ideología de los Estados Unidos, los gobiernos 
latinoamericanos reivindican un mayor peso político a nivel de la comunidad de Estados latinoamericanos estableciendo un balance a los proyectos hegemónicos. Bien que por el momento este contrapeso sea mayormente a nivel ideológico, es un primer paso para la reestructuración de medidas antidrogas.

A nivel de las perspectivas futuras, se puede considerar que estás dependen de un proceso social de reestructuración de identidades. En este sentido la posibilidad de establecer, a largo plazo, un nuevo régimen de políticas antidrogas por parte de los países latinoamericanos, aunque podría llegar a ser viable, sería el resultado de un largo proceso. Esto debido a la que reestructuración de identidades está ligada a políticas de poder que son renuentes al cambio. En primer lugar, "una vez formado, cualquier sistema social aparece ante cada uno de sus miembros como un hecho social objetivo que premia ciertos y disuade de mantener otros" (Wendt, 2005:16). Y en segundo lugar, "el cambio sistémico puede también ser impedido por actores interesados en mantener el papel relativamente estable de las identidades" (Wendt, 2005:16). En este sentido, la visión del sistema social sobre las drogas está ampliamente arraigada en la comunidad internacional. Como se mencionaba para el caso de Uruguay, aún hay numerosas preocupaciones sobre los problemas sociales y para la salud que pueden tener la legalización de las llamadas drogas blandas. Además, como vimos anteriormente, la adhesión a los diferentes convenios internacionales genera una serie de compromisos y expectativas ante la comunidad internacional que son difíciles de transgredir. También es importante agregar que las dinámicas de interdependencia entre los países hacen que sea difícil la aceptación de este tipo de medidas ante el temor que generan. Todo esto sin mencionar, que actores como las Naciones Unidas, y países como Estados Unidos o los miembros de la comunidad europea tienen herramientas de poder que pueden disuadir este tipo de acciones tomadas por los gobiernos Latinoamericanos.

\section{Conclusiones}

¿En qué medida la existencia de un cambio en la percepción del fenómeno de las drogas, ha generado un giro en las políticas destinadas al control de las mismas en América Latina? El fracaso de las políticas represivas implementadas en la segunda mitad del siglo XX en América Latina, enfocadas a la erradicación de la oferta ilegal de estupefacientes para así parar el flujo de los mismos hacia los países productores no tuvo los resultados esperados. 
En lugar de disminuir los problemas sociales asociados a su consumo, la violencia y el crimen siguieron siendo parte del panorama, a esto se agrega el empeoramiento de los problemas sanitarios, no solo en los países de Europa y Norte América sino también en el sur. Esto ha implicado una toma de conciencia por parte de los gobiernos latinoamericanos en la búsqueda de nuevas soluciones al problema, lo que ha implicado un cuestionamiento de su identidad como simpatizantes de la lucha antidrogas en términos de represión y cero tolerancia. Sin embargo, este esbozo de cambio de identidad está atado a una serie de dinámicas institucionalizadas formal e informalmente por la comunidad internacional, lo cual impide un cambio abrupto en la manera como se definen esas identidades y las repercusiones que esto puede tener en las acciones de los Estados. Por otra parte, estos giros en las políticas antidrogas ya han sido tomados en cuenta por organizaciones como la OEA, quien el pasado mayo publicó un informe sobre la política antidrogas en el que se incluye la despenalización como una medida estratégica para aliviar los problemas sanitarios generados por las drogas (LA Prensa, 2013). Desde este ángulo, se puede considerar que el liderazgo de estas iniciativas será llevado a cabo por organismos internacionales en una iniciativa de cooperación conjunta, más no por iniciativa individual de un Estado, debido a las sanciones sociales que esto puede implicar.

\section{Bibliografía}

BAGLEY, Bruce (2013). "Principales tendencias del siglo XXI en cuanto al crimen organizado, el narcotráfico y la democracia en la región”. En: Trans-pasando Fronteras, núm. 3), pp. 47-54. Cali, Colombia: Centro de Estudios Interdisciplinarios, Jurídicos, Sociales y Humanistas (CIES), Facultad de Derecho y Ciencias Sociales, Universidad Icesi.

CASTELLS, M. (1999). Globalización, identidad y Estado en América Latina. (P. d. desarrollo, Ed.) Temas de desarrollo sustentable, 21.

KAPLAN, Marcos (1993). "Tráfico de drogas en américa latina: emergencia, contexto internacional y dinámica interna”. En: Boletín Mexicano de Derecho Comparado, vol. XXVI, núm. 76, pp. 115-154. México D.F.: Universidad Nacional Autónoma de México

KEGLEY, C., y Wittkopf, E. (2006). World Politics: Trends and Transformation (Tenth ed.). Belmont, CA, United States of America: Thomson Wadsworth.

PARADA SUAREZ, D. C. (2006). "Entre la línea del apoyo y la intervención: ten- 
dencias de la ayuda militar de Estados Unidos a América Latina”. En: Zero. Universidad Externado de Colombia, pp. 54-57.

WENDT, A. (2005). La anarquía es lo que los estados hacen de ella: la construcción social de la politica de poder. En: Revista Relaciones internacionales, pp. 3-47. Madrid, España: Facultad de Derecho, Universidad Autónoma de Madrid

\section{Documentos de prensa}

AGUILAR VALENZUELA, R. (19 de noviembre de 2013). Cobertura de la violencia: lo que se debe hacer y comunicar. Recuperado el 2013 de noviembre de 2013, de El Economista: http://eleconomista.com.mx/columnas/columna-especial-politica/2013/11/19/ cobertura-violencia-lo-que-no-se-debe-hacer-comunicar

EL NUEVO HERALD (19 de noviembre de 2013). "Unión Europea preocupada por incremento de cocaína en Bolivia”. En: Nuevo Herald América Latina. Recuperado el 20 de noviembre de 2013, desde: http://www.elnuevoherald.com/2013/11/19/1618348/ue-preocupada-por-incremento-de.html

EL TIEMPO (11 de julio de 2012). “Unos hablan, otros hacen”. En: El Tiempo. Recuperado desde: http://www.eltiempo.com/archivo/documento/MAM-5553223

INFOBAE (15 de abril de 2012). El debate sobre las drogas pasa a la OEA. Recuperado el 15 de noviembre de 2013, desde: http://www.infobae.com/2012/04/15/1048411-eldebate-las-drogas-pasa-la-oea

INFOBAE (14 de noviembre de 2013). La posición de los países de América Latina sobre la ley de derribo de aviones. Recuperado el 18 de noviembre de 2013, desde: http://www. infobae.com/2013/11/14/1523708-la-posicion-los-paises-america-latina-la-ley-derriboaviones

ISACSON, A. (26 de julio de 2010). "Si el Plan Colombia es un éxito, ¿qué es un fracaso?”. En: Razón pública. Recuperado desde: http://www.razonpublica.com/index.php/ politica-y-gobierno-temas-27/1191-si-el-plan-colombia-es-un-exito-ique-es-un-fracaso. html\#sthash.MPio5fMz.dpuf

LA PRENSA. (17 de mayo de 2013). "Insulza entregó a Santos informe de la OEA sobre política antidrogas”. En: La Prensa. Recuperado desde: http://www.prensa.com/uhora/ mundo/insulza-presentacion-informe-de-drogas-oea-juan-manuel-santos/178195 
LOZANO, R. (17 de agosto de 2012). 'Legalización es una vía contra lacra de la droga': Fox. En: Portafolio. Recuperado el 18 de noviembre de 2013, desde: http://www.portafolio. co/detalle_archivo/DR-59379

MORALES SOLÁ, J. (17 de noviembre de 2013). "Un nuevo y dramático escándalo". En: La Nación. Recuperado el 18 de noviembre de 2013, de: http://www.lanacion.com. ar/1638986-un-nuevo-y-dramatico-escandalo

NIXON, R. (17 de junio de 1971). "Special Message to the Congress on Drug Abuse Prevention and Control". En: The American Presidency Project. Recuperado el 19 de noviembre de 2013, desde: http://www.presidency.ucsb.edu/ws/?pid=3048

OPPENHEIMER, Andrés (5 de agosto de 2013). “Análisis/El 'jolgorio’ de la legalización”. En: Portafolio. Recuperado el 15 de noviembre de 2013, desde: http://www.portafolio.co/detalle_archivo/DR-100315

REY, D. (15 de noviembre de 2013). "Iglesia argentina clama contra el narcotráfico". En: Nuevo Herald América Latina. Recuperado el 18 de noviembre de 2013, desde: http:// www.elnuevoherald.com/2013/11/15/1615322/iglesia-argentina-clama-contra.html

SAIZ, E. (19 de noviembre de 2013). "La DEA advierte de la expansión de los cárteles mexicanos en EE UU”. En: El País. Recuperado el 20 de noviembre de 2013, desde: http:// internacional.elpais.com/internacional/2013/11/19/actualidad/1384881221_534915.html

TERRA (18 de noviembre de 2013). "Seis detenidos en aeropuerto de Lima por intentar viajar con droga a España”. En: Terra Mundo. Recuperado el 18 de noviembre de 2013, desde: http://goo.gl/3sR9IH

UNIVISIÓN (20 de noviembre de 2013). "Falcao ficha por la ONU para sacarle 'tarjeta roja a las drogas"”. En: Univisión Noticias. Recuperado el 20 de noviembre de 2013, desde: http://feeds.univision.com/feeds/article/2013-11-20/falcao-ficha-por-la-onu?refPath=/noticias/america-latina/colombia/

\section{Fuentes normativas}

HOPENHAYN, M. y Arriagada, I. (Octubre de Octubre 2000). Producción, tráfico y consumo de drogas en América Latina. Santiago de Chile: División de Desarrollo Social CEPAL-ECLAC y Naciones Unidas.

JUNTA INTERNACIONAL DE FISCALIZACIÓN DE ESTUPEFACIENTES (1991). 
ler Informe de la Junta Internacional de Fiscalización de Estupefacientes correspondiente a 1999. Nueva York: Publicaciones Naciones Unidas.

JUNTA INTERNACIONAL DE FISCALIZACIÓN DE ESTUPEFACIENTES (2013). Informe. Nueva York: Publicaciones Naciones Unidas.

ONU (1961). "Convención única de 1961 sobre estupefacientes. Organización de las Naciones Unidas". En: UNODC. Recuperado el 15 de noviembre de 2013, desde: http:// www.unodc.org/pdf/convention_1961_es.pdf

ONU (1972). "Convention on psychotropic substances. Organización de las Naciones Unidas”. En: UNODC. Recuperado el 15 de noviembre de 2013, desde: http://www.unodc. org/pdf/convention_1971_en.pdf

ONU (1988). Convención de las naciones unidas contra el tráfico ilicito de estupefacientes y sustancias sicotrópicas. Organización de las Naciones Unidas. Recuperado el 15 de noviembre de 2013, de unodc: https://www.unodc.org/pdf/convention_1988_es.pdf

OEA (14-15 de Abril de 2012). Comunicado de los jefes de estado y de gobierno sobre la realización de la conferencia internacional de ministros de relaciones exteriores y jefes de organismos nacionales especializados contra el problema mundial de las drogas. Recuperado el 19 de noviembre de 2013, de Organización de los Estados Americanos: http:// www.summit-americas.org/SIRG/2012/041412/reunion_drugs_es.pdf

SECRETARÍA GENERAL (2013). El problema de las drogas en las Américas. Organización de Estados Americanos.

SECRETARÍA OEA. (s.f.). Cumbres de las Américas. Obtenido de Organización de los Estados Americanos: http://www.summit-americas.org/sextacumbre.htm 